\title{
易电离元素 $\mathrm{Na}$ 和 $\mathrm{K}$ 对水工质脉冲等离子体 推进器性能的影响
}

\author{
朱平 ${ }^{(12}{ }^{*}$, 侯丽雅 ${ }^{(1)}$, 章维一 ${ }^{(1)}$ \\ (1) 南京理工大学机械工程学院, 南京 210094; \\ (2) 中北大学电子测试技术国家重点实验室, 太原 030051 \\ *E-mail: hyuanpeng@sohu.com
}

收稿日期: 2010-03-09; 接受日期: 2010-06-23

国家自然科学基金(批准号：60778029)、教育部博士学科点专项科研基金(批准号：20060288005)和江苏省自然科学基金(批准号: BK2007209)资助项目

\begin{abstract}
摘要针对水工质脉冲等离子体推进器的高电阻造成推功比低这一情况, 本文提出在水工 质中添加低电离势的易电离元素 $\mathrm{Na}$ 和 $\mathrm{K}$ 以提高其性能, 并通过放电电流测量和等离子体发射 光谱分析实验进行验证. 实验研究表明: $\mathrm{Na}$ 和 $\mathrm{K}$ 对脉冲等离子体推进器性能的提高有一定影 响. $\mathrm{NaCl}$ 和 $\mathrm{KCl}$ 水溶液工质与水工质相比, 具有较低的总电阻和较高的推功比和比冲. 其中, $\mathrm{NaCl}$ 水溶液工质对脉冲等离子体推进器性能的影响比 $\mathrm{KCl}$ 水溶液工质略高. 等离子体发射光 谱分析与放电电流测量实验一致. $\mathrm{Na}$ 和 $\mathrm{K}$ 致使水工质的等离子体发射光谱信号强度得到增强.
\end{abstract}

关键词 脉冲等离子 体推进器 水工质 易电离元素
近年来, 脉冲等离子体推进器 (Pulsed Plasma Thruster, PPT) 因其结构简单、可靠性高, 非常适合于 微型飞行器, 而受到广泛的关注. 常见的 PPT 使用 Teflon 作为工质, 然而 Teflon PPT 存在一些问题 ${ }^{[1 ~ 3]}$, 如主放电期间的低速蒸汽和大粒子的发散造成的推 进性能低、Teflon 烧蚀后产生的碳和氟污染飞船上的 设备、放电电流密度的不均匀造成系统的比冲降低等. 由于水与 Teflon 相比, 能减少羽流污染, 并具有更高 的比冲. 因此, 一些研究人员开始研究基于水工质的 $\mathrm{PPT}^{[4,5]}$. 然而, 在推功比方面, 研究表明水与 Teflon 相比其值较低. 比如: Carsten ${ }^{[6]}$ 的水和 Teflon PPT 的 放电对比测试实验就表明水工质 PPT 的总电阻比 Teflon PPT 大, 造成水工质 PPT 的推功比小于 Teflon PPT. Hiroyuki等人 ${ }^{[7]}$ 的推力测试实验再一次表明水
工质 PPT 的推功比小于 Teflon PPT. 水工质 PPT 的高 电阻是造成其推功比低的主要原因, 如何降低基于 水工质 PPT 的总电阻来以提高推功比是值得研究的. 因此, 本文提出通过在水工质中添加低电离势的易 电离元素 $\mathrm{Na}$ 和 $\mathrm{K}$ 来促进电子碰撞激发, 增强水分子 的电离, 实现减小总电阻的目的, 达到提高推功比的 效果.

\section{1 易电离元素对等离子体电磁加速影响分析}

根据文献[8]所述，等离子体电磁加速产生的冲 量和比冲分别表示为

$$
I_{\mathrm{EM}}=\int_{0}^{\tau} F_{\mathrm{EM}} \mathrm{d} t \cong \frac{1}{2} L^{\prime} \frac{E_{0}}{R_{\mathrm{all}}},
$$




$$
I_{\mathrm{SP}}=I_{\mathrm{EM}} /(\Delta m \cdot g),
$$

式中 $L^{\prime}$ 是单位电感值, $E_{0}$ 是储能电容存储能量, $R_{\mathrm{all}}$ 是 PPT 的总电阻, $\Delta m$ 是消耗的平均工质质量, $g$ 是重力 加速度.

(1)式中, 总电阻 $R_{\mathrm{all}}$ 是由 PPT 外部电路中的电 容、电极和触发电路的等效电阻 $R_{\mathrm{c}}$ 和等离子体的等 效电阻 $R_{\mathrm{p}}$ 以及作电磁功的等效电阻 $R_{\mathrm{EM}}$ 组成, 即

$$
R_{\mathrm{all}}=R_{\mathrm{c}}+R_{\mathrm{p}}+R_{\mathrm{EM}} .
$$

从(1)式可以看出, 非常小的总电阻导致很高的 推功比, 并且由等离子体电磁加速制造的冲量并不 依赖于工质质量. 如果想提高冲量, 必然要增加存储 能量值来强化工质的热电离, 或者是降低总电阻值 $R_{\text {all }}$. 提高 PPT 性能的传统作法是对存储能量和电离 腔的结构进行优化, 或者是对存储能量和工质进给 率进行优化 ${ }^{[9 \sim 11]}$. 对于水工质 PPT 来说, 高电阻是造 成其推功比低的主要原因, 增加存储能量值仅仅影 响的是等离子体密度, 然而造成高电阻的低电子温 度 $T_{\mathrm{e}}$ 或低电子碰撞频率并没有得到实质性的提高(充 分电离的等离子体中的电阻率是与 $\left(k T_{\mathrm{e}}\right)^{3 / 2}$ 成比率, 且并不依赖于等离子体密度). 基于减少电阻值的必 要性, 我们需要选择一种添加剂添加到水工质中以 提高水分子的电离率, 增强电子密度, 提高电子碰撞 频率, 实现减少总电阻的目的. 由于低电离势的易电 离元素(如: $\mathrm{Li} 、 \mathrm{Na} 、 \mathrm{~K}$ 和 $\mathrm{Ca}$ 等碱金属附随物)具有使 母液中的原子或分子激发和离解条件变化、促进电子 碰撞激发、改善等离子体的热传导等 ${ }^{[12 \sim 15]}$ 作用. 因此, 我们推断电离势小于水分子的 $\mathrm{Na}$ 和 $\mathrm{K}$ 适合作为添加 剂. $\mathrm{Na}$ 和 $\mathrm{K}$ 电离产生的额外电子将会改变等离子体 的整个激励环境, 水分子的电离将得到促进, 致使等 离子体中的电子密度得到增强, 电子碰撞频率得到 提高.

本文通过放电电流测量结合等离子体发射光谱 分析实验来研究易电离元素 $\mathrm{Na}$ 和 $\mathrm{K}$ 对水工质 $\mathrm{PPT}$ 性能的影响.

\section{2 实验}

\section{1 推进器结构和工质的制备}

水工质 PPT 的结构主要由同轴阴极、实心阳极、 喷嘴、火花塞以及水蒸气供给通道组成, 如图 1 所示. 同轴阴极的内径为 $7 \mathrm{~mm}$, 长 $24 \mathrm{~mm}$, 材料为紫铜. 实
心阳极直径为 $2.4 \mathrm{~mm}$, 材料为针铇. 阴极和阳极是 由陶瓷构件隔离. 火花塞安装在阴极上.

亚沸蒸馏水被选作工质. 钠 $\mathrm{Na}$ 和钾 $\mathrm{K}$ 被选作溶 解物添加到水工质中. $\mathrm{NaCl}$ 用亚沸蒸馏水溶解，配制 成 $\mathrm{NaCl}$ 水溶液. $\mathrm{KCl}$ 用亚沸蒸馏水溶解，配制成 $\mathrm{KCl}$ 水溶液. 本次实验中，碱金属氯化物和亚沸蒸馏水的 摩尔百分数在 $0.5 \%$ 2.2\%范围内时, 谱线强度显著增 强. 当加入的碱金属氯化物超出这个范围, 谱线强度 则有降低的趋势，甚而至于产生自蚀现象. 表 1 列出 了 $\mathrm{H}_{2} \mathrm{O}, \mathrm{Na}$ 和 $\mathrm{K}$ 的电离电势及其最佳水溶液的摩尔 百分数.

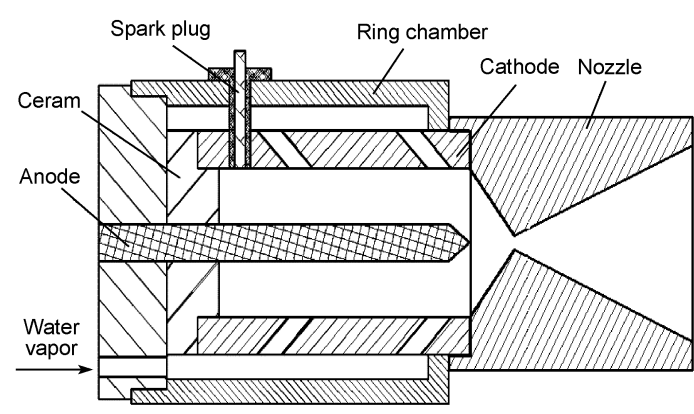

图 1 PPT 结构示意图

表 $1 \mathrm{H}_{2} \mathrm{O}, \mathrm{Na}$ 和 $\mathrm{K}$ 的电离电势及其水溶液浓度

\begin{tabular}{ccc}
\hline & $\begin{array}{c}\text { Ionization } \\
\text { potential }(\mathrm{eV})\end{array}$ & $\begin{array}{c}\text { Solution } \\
\text { concentration }(\%)\end{array}$ \\
\hline $\mathrm{H}_{2} \mathrm{O}$ & $12.7\left(\rightarrow \mathrm{H}_{2} \mathrm{O}^{+}\right)$ & - \\
$\mathrm{Na}$ & $5.1\left(\rightarrow \mathrm{Na}^{+}\right)$ & 2 \\
$\mathrm{~K}$ & $4.3\left(\rightarrow \mathrm{K}^{+}\right)$ & 1.2 \\
\hline
\end{tabular}

\section{2 测试系统}

测试实验在气压 $10^{-2} \mathrm{~Pa}$ 的近似真空环境下进行. 三种工质均采用超声波雾化后进入电极空间. 水蒸 气流量为 $5 \mu \mathrm{g} / \mathrm{s}$. 放电电流使用示波器 TDS2004B、 高压探头及罗氏线圈进行测量. 等离子体的光谱使 用单色仪(SBP500)进行检测．等离子体的发射光信 号透过双凸透镜聚焦进入光纤, 然后导入单色仪. 该 单色仪具有 $500 \mathrm{~mm}$ 焦距, 分辨率 $0.05 \mathrm{~nm}$, 倒线色散 $1.7 \mathrm{~nm} / \mathrm{mm}$, 重复性 $0.05 \mathrm{~nm}$, 最小步距 $0.0025 \mathrm{~nm}$. 狭 缝宽度设置为 $100 \mu \mathrm{m}$. 曝光时间的设置需要比 PPT 的放电时间长，因此设置为 $2 \mathrm{~s}$. 被扫描的光谱波长 为 300 800 $\mathrm{nm}$. 光谱波长和辐射率使用氩放电管和 标准源校准. 


\section{3 结果分析}

\section{1 电路参数的测量和分析}

在(3)式中, 等效电阻 $R_{\mathrm{c}}$ 是一个与外部电路的结 构参数相关的等效值, 其值是不会受到存储能量和 易电离元素干扰而变化, 因而把它近似为一个常数. 等效为存储能量转化为焦耳热部分的电阻 $R_{\mathrm{p}}$ 和作电 磁功的等效电阻 $R_{\mathrm{EM}}$, 由于容易受到存储能量和等离 子体中带电粒子的影响而变化, 因此, 在这里被设定 为变量. 根据以上的分析, 可以这样认为: 总电阻值 $R_{\text {all }}$ 的变化实质是等效电阻 $R_{\mathrm{p}}$ 和 $R_{\mathrm{EM}}$ 的变化. 外部电 路中的电容、电极和触发电路的等效电阻 $R_{\mathrm{c}}$ 和单位 电感值 $L^{\prime}$ 是通过拟合在大气压下的水工质 PPT 短路 时的放电电流得到. 具体的方法是在电极间施加 $1000 \mathrm{~V}$ 的电压, 然后用铜导线短路, 测量出放电电流. 根据放电电流拟合出等效电阻为 $R_{\mathrm{c}}=62.50 \mathrm{~m} \Omega$. 通过 移动铜导线短路位置, 估算出单位电感值为 $L^{\prime}=1.92$ $\mathrm{nH} / \mathrm{mm}$. 等效电阻值 $R_{\mathrm{p}}+R_{\mathrm{EM}}$ 就可以通过总电阻值 $R_{\mathrm{all}}$ 减去 $R_{\mathrm{c}}$ 得到.

基于三种类型工质 $\left(\mathrm{H}_{2} \mathrm{O}, \mathrm{KCl}\right.$ 和 $\mathrm{NaCl}$ 水溶液)的 PPT 在储存能量为 $4.86 \mathrm{~J}$ 时的放电电流测量值如图 2 所示. 从图 2 可以看出基于水工质的放电电流峰值明 显低于基于 $\mathrm{NaCl}$ 和 $\mathrm{KCl}$ 水溶液工质的放电电流峰值, 尤其在放电的初始相位, 水工质的放电电流曲线图 呈现出更多的不规则性. 这表明工质中添加 $\mathrm{Na}$ 和 $\mathrm{K}$ 后, 等离子体的等效电阻值有明显降低, 与此同时还 增强了 PPT 工作时的稳定性.

基于三种类型工质 $\left(\mathrm{H}_{2} \mathrm{O}, \mathrm{KCl}\right.$ 和 $\mathrm{NaCl}$ 水溶液)的 的 PPT 在不同存储能量下的总电阻列于表 2. 表 2 表 明 $\mathrm{NaCl}$ 和 $\mathrm{KCl}$ 水溶液工质的总电阻值与水工质相比 都有所下降, 降幅的平均值分别为 $3.48 \%$ 和 $2.56 \%$. $\mathrm{NaCl}$ 水溶液工质的总电阻值比 $\mathrm{KCl}$ 水溶液工质略低. 在存储能量大于 $6.40 \mathrm{~J}$ 后, 两种水溶液工质的总电阻 趋于一个常数. 可以看出两种水溶液工质对降低 PTT 的总电阻值是有限的, 并且降幅与存储能量值的 范围存在一定的优化关系.

基于三种类型工质 $\left(\mathrm{H}_{2} \mathrm{O}, \mathrm{KCl}\right.$ 和 $\mathrm{NaCl}$ 水溶液 $)$ 的 PPT 在不同存储能量下的推功比列于表 3. 推功 比的计算是根据(1)式得到的冲量值除以存储能量值 得到. 表 3 表明, $\mathrm{NaCl}$ 和 $\mathrm{KCl}$ 水溶液工质的推功比 比水工质高, 并且 $\mathrm{NaCl}$ 水溶液工质的推功比比 $\mathrm{KCl}$ 水溶液工质略高, 两者的平均增幅分别为 $3.81 \%$ 和

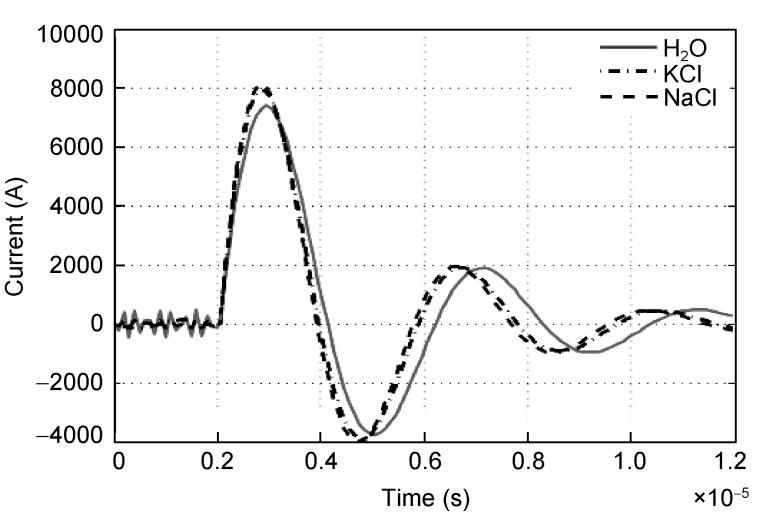

图 2 基于 $\mathrm{H}_{2} \mathrm{O}, \mathrm{KCl}$ 和 $\mathrm{NaCl}$ 水溶液工质的 PPT 放电电流值

表 2 不同存储能量下的等效总电阻

\begin{tabular}{cccc}
\hline \multirow{2}{*}{ Stored energy $(\mathrm{J})$} & \multicolumn{3}{c}{ Total resistance $(\mathrm{m} \Omega)$} \\
\cline { 2 - 4 } & $\mathrm{H}_{2} \mathrm{O}$ & $\mathrm{NaCl}$ & $\mathrm{KCl}$ \\
\hline 3.56 & 89.11 & 86.03 & 87.21 \\
4.86 & 87.07 & 85.15 & 86.32 \\
5.18 & 85.98 & 82.89 & 83.01 \\
6.00 & 85.01 & 82.06 & 83.10 \\
6.40 & 85.01 & 81.04 & 81.99 \\
\hline
\end{tabular}

表 3 不同存储能量下的推功比

\begin{tabular}{cccc}
\hline \multirow{2}{*}{ Stored energy $(\mathrm{J})$} & \multicolumn{3}{c}{ Thrust power ratio $(\mu \mathrm{Ns} / \mathrm{J})$} \\
\cline { 2 - 4 } & $\mathrm{H}_{2} \mathrm{O}$ & $\mathrm{NaCl}$ & $\mathrm{KCl}$ \\
\hline 3.56 & 10.78 & 11.16 & 11.03 \\
4.86 & 11.04 & 11.36 & 11.16 \\
5.18 & 11.20 & 11.57 & 11.57 \\
6.00 & 11.29 & 11.78 & 11.57 \\
6.40 & 11.29 & 11.85 & 11.71 \\
\hline
\end{tabular}

$2.58 \%$.

在存储能量 $6.40 \mathrm{~J}$ 时, $\mathrm{NaCl}$ 水溶液工质的推功比 增幅达到 $4.96 \%$. 可以看出推功比的增幅趋势强烈依 赖于能量的增加. 从比冲的角度来看, 根据(2)式的 定义, 在存储能量和消耗的平均工质质量不变的前 提下, 易电离元素的合理添加使比冲也能得到与推 功比增幅相同的提高.

\section{2 光谱分析}

图 3 显示了 PPT 在储存能量为 $4.86 \mathrm{~J}$ 时三种类 型工质 $\left(\mathrm{H}_{2} \mathrm{O}, \mathrm{KCl}\right.$ 和 $\mathrm{NaCl}$ 水溶液)的等离子体发射光 

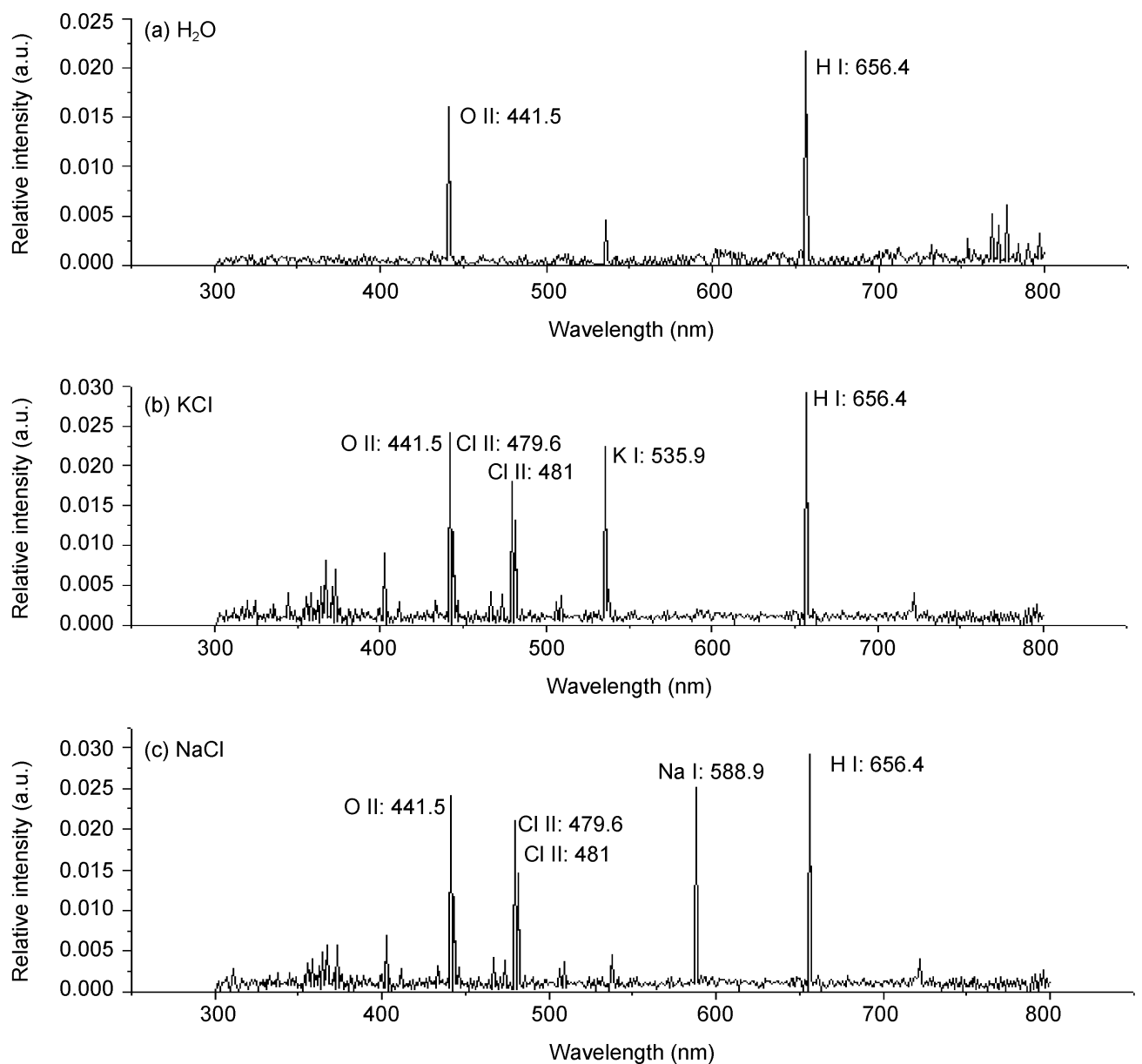

图 $3 \mathrm{H}_{2} \mathrm{O}(\mathrm{A}), \mathrm{KCl}(\mathrm{B})$ 和 $\mathrm{NaCl}(\mathrm{C})$ 水溶液的等离子体发射光谱

谱. $\mathrm{KCl} 、 \mathrm{NaCl}$ 水溶液工质的等离子体中的氢原子 $(\mathrm{H} \mathrm{I})$ 、钠原子 $(\mathrm{Na} \mathrm{I})$ 、钾原子 $(\mathrm{K} \mathrm{I})$ 通过与中国基础科 学数据中心的原子发射光谱数据库(BSDC)进行比对 识别出. 此外, 被电离的氧离子 $(\mathrm{O} I \mathrm{II})$ 、氯离子 $(\mathrm{Cl} \mathrm{II})$ 的发射谱线通过与美国国家标准与技术研究院原子 吸收和发射性能数据库(NIST)进行比对也被识别出. 由于钠离子 $(\mathrm{Na} I \mathrm{II})$ 、钾离子 $(\mathrm{K} \mathrm{II})$ 和氯原子 $(\mathrm{Cl} I)$ 的发 射谱线超出单色仪的扫描范围, 因此没有观察到这 些谱线. 从光谱图中可以看出被易电离元素 $\mathrm{Na}$ 和 $\mathrm{K}$ 干扰的氢原子 $(\mathrm{H} \mathrm{I})$ 、氧离子 $(\mathrm{O}$ II $)$ 的发射光谱强度明 显比 $\mathrm{H} 2 \mathrm{O}$ 强, 并且这两种光谱图有相似的谱线特征. 正如前言所介绍, 钠原子 $(\mathrm{Na} I)$ 和钾原子(K I)电离产 生的额外电子促进了电子碰撞激发, 增强了水分子 的电离, 并且使等离子体中的电场分布更加均匀, 从 而影响了光谱信号强度.

\section{4 结论}

通过放电电流测量和等离子体发射光谱分析实 验研究了低电离势的易电离元素 $\mathrm{Na}$ 和 $\mathrm{K}$ 对水工质 PPT 性能的影响. 放电电流测量实验研究表明, $\mathrm{Na}$ 和 $\mathrm{K}$ 对水工质 PPT 总电阻的减少有一定影响. $\mathrm{NaCl}$ 和 $\mathrm{KCl}$ 水溶液工质与水工质相比, PPT 的总电阻都有所 降低, 致使推功比和比冲都得到提高.

其中, $\mathrm{NaCl}$ 水溶液工质对 PPT 性能的影响比 $\mathrm{KCl}$ 水溶液工质略高. 然而, 两种水溶液工质对 PPT 总电 阻的影响是有限的, 并且 PPT 总电阻的降幅与存储 能量值的范围存在一定的优化关系. 等离子体发射 光谱分析与放电电流测量实验一致, 光谱分析表明, 钠原子和钾原子电离产生的额外电子促进了电子碰 撞激发, 增强了水分子的电离, 致使 $\mathrm{NaCl}$ 和 $\mathrm{KCl}$ 水 溶液工质的等离子体光谱信号强度得到增强. 


\section{参考文献}

1 Christopher D R, Mark E C, Mattick A T. Pulsed plasma thruster system for microsatellites. J Spacecraft Rockets, 2005, 42(1): 161-170

2 Uezu J J, IioJ P, Yukiya K, et al. Study on pulsed plasma thruster configuration to expand impulse bit range. In: 29th International Electric Propulsion Conference. IEPC. 2005. 234

3 Spanjers G G, Lotspeich J S, McFall K A, et al. Propellant losses because of particulate emission in a pulsed plasma thruster. J Propul Power, 1998, 14(4): 554-559

4 Markusic T E, Kurt A P, Choueiri E Y, et al. Ablative z-pinch pulsed plasma thruster. J Propul Power, 2005, 21(3): 392-405

5 Hiroyuki K, Kakami A, Furuta Y, et al. Liquid propellant pulsed plasma thruster. In: 28th International Electric Propulsion Conference. IEPC. 2003. 087

6 Carsten A S. Investigation of thrust mechanisms in a water fed pulsed plasma thruster. Dissertation of Doctoral Degree. Columbus: Ohio State University, 2003. 68-70

7 Hiroyuki K, Yohei F, Kimiya K, et al. A pulsed plasma thruster using water as the propellant. In: 40th AIAA/ASME/SAE/ASEE Joint Propulsion Conference and Exhibition. AIAA. 2004. 3460

8 Jahn R G. Physics of Electric Propulsion. New York: McGraw-Hill, 1968. 12-13

9 侯大立, 赵万生, 康小明. 脉冲等离子体推力器的性能分析. 推进技术, 2008, 29(3): 377-380

10 刘莉莉, 邓建新, 皇攀凌, 等. $\mathrm{SiC}(\mathrm{W}, \mathrm{Ti}) \mathrm{C}$ 梯度陶瓷喷嘴的设计与制备. 中国科学 $\mathrm{E}$ 辑：技术科学, 2007, 37(7): 857-865

11 Harada N, Kien L C, Tashiro T. Closed cycle mhd generator using He/Xe working plasma. In: 33rd Plasma Dynamics and Lasers Conference. 2002. 2144

12 Alan M P, Nick P L, John C V, et al. Salt effects on ion formation in desorption mass spectrometry: An investigation into the role of alkali chlorides on peak suppression in time-of-flight-secondary ion mass spectrometry. Anal Chem, 2009, 81(3): 1040-1048

13 严建华, 戴尚莉, 李晓东, 等. 气液两相滑动弧放电中自由基的光谱研究. 光谱学与光谱分析, 2008, 28(8): 1851-1855

14 Song W D. Active electrospray ionization for efficient electric thrusters. In: 40th AIAA/ASME/SAE/ASEE Joint Propulsion Conference and Exhibition. 2004. 3942

15 Wang C C, Subrata R. Microscale plasma actuators for improved thrust density. J Appl Phys, 2009, 17(7): 013310-013317 